\title{
Flow and heat transfer in cavities between rotor and stator disks
}

\author{
Gian Paolo Beretta ${ }^{a, *}$, Enrico Malfa ${ }^{b, 1}$ \\ ${ }^{a}$ INFM, Università di Brescia, via Branze 38, 25123 Brescia, Italy \\ b ABB Ricerca SpA, viale Edison 50, Sesto San Giovanni, Milano, Italy
}

Received 19 March 2001; received in revised form 20 January 2003

\begin{abstract}
The paper discusses the aerodynamic effects in a rotor-stator arrangement where rotor-induced air flow is important in the gap between rotor and housing bases. The rotor is adiabatic whereas the housing surfaces are assumed isothermal. For the case of a closed housing, the empirical correlations available in the literature for the estimate of moment coefficients due to aerodynamic effects under similar geometrical and kinematic conditions are compared with the results of CFD simulations. The numerical results on moment coefficients, mechanical power dissipation, and velocity fields are in satisfactory agreement. In order to evaluate temperature fields and heat fluxes, because no empirical correlations were found for the adiabatic-rotor/isothermal-stator conditions of interest, a semi-empirical model was developed, based on mass and angular momentum balances and the Reynolds analogy. Numerical results and approximate estimates of temperature distribution on the rotor surfaces are in reasonable agreement, also for the case of a housing open to radial flow through the gap.
\end{abstract}

(c) 2003 Elsevier Science Ltd. All rights reserved.

\section{Introduction}

Fluid flow and heat transfer in enclosed rotor-stator systems have been the subject of a large number of studies, mostly for gas turbine applications [1], where air flow in the wheel space between turbine disk and adjacent stationary casing is used for cooling purposes. In these applications, frictional heating and compressive work are important due to high-rotational speeds. Another area where high-rotational speeds are becoming less unusual is in the design of advanced electric motors [2].

Daily and Nece [3] established empirical correlations for aerodynamic generated torque valid for turbulent flow with no radial outflow within a closed rotor-stator

\footnotetext{
${ }^{*}$ Corresponding author.

E-mail addresses: beretta@ing.unibs.it (G.P. Beretta), enrico.malfa@c-s-m.it (E. Malfa).

${ }^{1}$ Present Address: Centro Sviluppo Materiali, SpA, piazza Caduti 6 Luglio 1944, Bergamo, Italy.
}

system with rotational Reynolds numbers $R e_{\phi}$ up to $10^{7}$ and Couette Reynolds numbers $R e_{\text {Couette }}$ up to $2 \times 10^{6}$. Kreith [4] later proposed slight corrections to the same correlations.

In the present work, the Kreith-Daily-Nece correlations are used to evaluate the flow-induced torque in the rotor-stator disks and to validate a numerical study performed with the commercial code Fluent on a case with $R e_{\phi}$ up to $2.6 \times 10^{6}$ and $R e_{\text {Couette }}$ up to $4 \times 10^{4}$. The results are discussed in Section 2.1.

However in high-rotational-speed electric-motor applications not only the flow field and aerodynamic power dissipation are of interest but also temperature distribution and heat transfer between rotor and stator.

In the present case, the rotating surface is assumed to be approximately adiabatic whereas the stationary surface is cooled and assumed to be maintained at an approximately uniform temperature. Since the heat transfer experimental data and correlations for Nusselt numbers are available in the literature for different boundary conditions, i.e., heated-rotor/cooled-stator 


\begin{tabular}{|c|c|c|c|}
\hline \multicolumn{4}{|c|}{ Nomenclature } \\
\hline$a$ & stator inner radius & $W$ & shaft power \\
\hline$b$ & disk radius & $w^{*}$ & friction velocity \\
\hline$C_{\mathrm{M}}$ & moment coefficient & $x$ & dimensionless radial coordinate $(r / b)$ \\
\hline$C_{\mathrm{n}}$ & power law coefficient & $x_{\mathrm{a}}$ & dimensionless stator inner radius $(a / b)$ \\
\hline$c_{\mathrm{p}}$ & specific heat & $z$ & axial coordinate \\
\hline$C_{\mathrm{w}}$ & dimensionless radial flow rate & $z^{+}$ & dimensionless wall distance $(z \sqrt{\tau / \rho} / v)$ \\
\hline$G$ & dimensionless clearance $(s / b)$ & $\beta$ & center gap dimensionless swirl $\left(\beta_{1 / 2}\right)$ \\
\hline$H$ & enthalpy & $\beta_{\gamma}$ & dimensionless swirl at dimensionless axial \\
\hline$k$ & thermal conductivity & & coordinate $\gamma$ \\
\hline$k_{\mathrm{s}}$ & effective roughness height & $\Gamma$ & dimensionless swirl $\left(v_{\phi} / \Omega r\right)$ \\
\hline$k_{\mathrm{T}}$ & turbulent kinetic energy & $\mu$ & viscosity \\
\hline K & kelvin (unit of absolute temperature) & $v$ & kinematic viscosity \\
\hline$M$ & torque & $\Omega$ & angular speed \\
\hline$n$ & power law inverse exponent & $\rho$ & density \\
\hline$q$ & heat flux & $\tau$ & shear stress \\
\hline$Q$ & thermal power exchange & $\Theta$ & dimensionless total enthalpy \\
\hline & radial coordinate & \multicolumn{2}{|c|}{ Subscripts } \\
\hline$r_{\mathrm{o}}$ & shaft radius & $\mathrm{c}$ & center gap \\
\hline $\operatorname{Re}_{\phi}$ & rotational Reynolds number $\left(\Omega b^{2} / v\right)$ & eff & effective \\
\hline $\operatorname{Re}_{\text {Couette }}$ & Couette Reynolds number $(\Omega b s / v)$ & ell & enlective \\
\hline$R e_{z}$ & wall-distance-based Reynolds number & $\begin{array}{l}\mathrm{O} \\
\mathrm{r}\end{array}$ & $\begin{array}{l}\text { rotor } \\
\text { radial }\end{array}$ \\
\hline$P r$ & $\begin{array}{l}\left(z \sqrt{ } k_{T} / v\right) \\
\text { Prandtl number }\end{array}$ & s & stator \\
\hline$s$ & axial gan clearance & $z$ & axial \\
\hline $\begin{array}{l}S \\
T\end{array}$ & $\begin{array}{l}\text { axial gap clearance } \\
\text { temperature }\end{array}$ & $\phi$ & tangential \\
\hline $\begin{array}{l}1 \\
v\end{array}$ & $\begin{array}{l}\text { temperature } \\
\text { flow velocity component }\end{array}$ & & \\
\hline
\end{tabular}

[5-13], they cannot be readily extrapolated to determine the temperature distributions on the rotor surface in the adiabatic-rotor/cooled-stator case.

Therefore, the thermal aspects have been approached using a numerical study validated by verifying the Reynolds analogy [14, p. 255] between swirl velocity and total enthalpy fields for the case of a closed system with adiabatic rotor, isothermal stator, no radial outflow and unit Prandtl number. Discussion and results of this validation are given in Section 3.

Finally, because no empirical correlations for moment coefficients and Nusselt numbers were found for the gap in presence of radial outflow for the rotor-stator conditions of interest, we developed and validated a semi-empirical method, discussed in Section 4, based on mass and angular momentum balances and on the Reynolds analogy, for an approximate estimate of the flow field and the temperature distribution on the rotating disk surface.

The Reynolds analogy yields results that are at first sight somewhat counterintuitive, in that the presence of radial flow has little influence on the adiabatic disk temperature distribution, however, the predictions are confirmed by the numerical study.

\section{Aerodynamic power dissipation-empirical correlations}

\subsection{Cavity with no radial flow}

Based on the geometrical parameters presented in Fig. 1, the rotor-stator configuration of reference in this study is defined by the following parameters
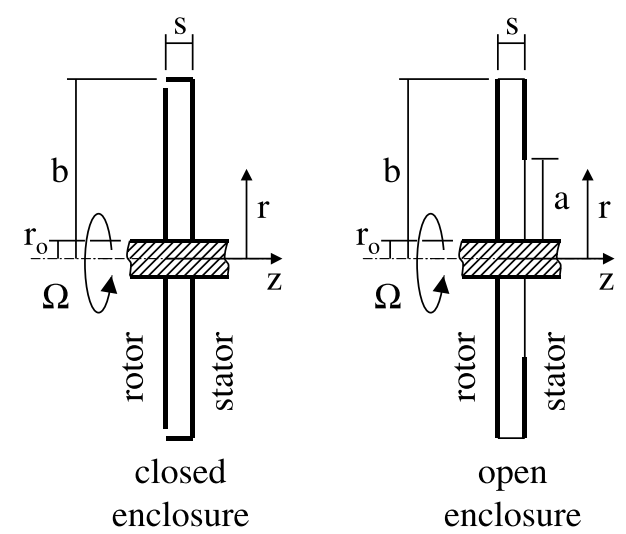

Fig. 1. Sketch of closed and open rotor-stator enclosures. 


$$
\begin{aligned}
\Omega & =85000 \mathrm{rpm} \\
b & =70 \mathrm{~mm} \\
s & =1 \mathrm{~mm} \\
r_{\mathrm{o}} & =10.4 \mathrm{~mm} \\
a & =10.4 \mathrm{~mm} \text { (closed enclosure) } \\
& =11.4 \mathrm{~mm} \text { (open } 1 \mathrm{~mm}) \\
& =29.4 \mathrm{~mm} \text { (open } 19 \mathrm{~mm}) \\
& =49 \mathrm{~mm} \text { (open } 38.6 \mathrm{~mm})
\end{aligned}
$$

$G=\frac{s}{b}=0.0143$

where $s$ is the axial gap clearance, $G$ is the dimensionless clearance, $r_{\mathrm{o}}$ is the shaft radius and $a$ the stator inner radius (for further reference we indicated here also the values of $a$ for the cases considered later in which the gap is open to radial outflow). For the closed enclosure case the lateral surface is stationary.

For air at $300 \mathrm{~K}\left(v=1.658 \times 10^{-5}\right)$ these data correspond to

$$
\begin{aligned}
& R e_{\phi}=\frac{\Omega b^{2}}{v}=2.63 \times 10^{6} \\
& R e_{\text {Couette }}=\frac{\Omega b s}{v}=R e_{\phi} G=37600
\end{aligned}
$$

At $400 \mathrm{~K}\left(v=2.734 \times 10^{-5}\right)$

$R e_{\phi}=\frac{\Omega b^{2}}{v}=1.595 \times 10^{6}$

$R e_{\text {Couette }}=\frac{\Omega b s}{v}=\operatorname{Re}_{\phi} G=22800$

The mechanical power dissipation is given by

$$
\begin{aligned}
& W=M \Omega=\frac{1}{2} C_{\mathrm{M}} \rho \Omega^{3} b^{5} \\
& M=\int_{a}^{b} r \tau_{\mathrm{o}} 2 \pi r \mathrm{~d} r \\
& C_{\mathrm{M}}=\frac{M}{\frac{1}{2} \rho \Omega^{2} b^{5}}=4 \pi \int_{x_{\mathrm{a}}}^{1} x^{2} \frac{\tau_{\mathrm{o}}}{\rho \Omega^{2} b^{2}} \mathrm{~d} x
\end{aligned}
$$

where $\tau_{\mathrm{o}}$ is the tangential stress component on the rotating disk surface, $M$ is the torque, $C_{\mathrm{M}}$ the moment coefficient, $x=r / b$ the dimensionless radial coordinate and $x_{\mathrm{a}}=a / b$.

Theoretical and experimental work on laminar to turbulent transition in the flow on a free rotating disk show that the relevant parameter is $x^{2} R e_{\phi}$.

On a highly polished disk, departure from laminar flow has been found to begin at about $x^{2} R e_{\phi}=3.1 \times 10^{5}$ and fully established turbulent flow to occur for $x^{2} R e_{\phi}>7 \times 10^{5}[1$, p. 67]. However, on a roughened disk or in the presence of external disturbances, transition can occur at values of $x^{2} R e_{\phi}$ well below $2.2 \times 10^{5}$ so that even at Reynolds numbers as low as $\operatorname{Re}_{\phi}=2.1 \times 10^{5}$ the moment coefficient may show no sign of transitional flow.

For a closed rotor-stator enclosure, the experimental work of Daily and Nece [3] as later recorrelated by Kreith [4] shows that the flow is turbulent in the entire enclosure for

$R e_{\phi}>\left(\frac{\pi}{0.036}\right)^{4 / 3} G^{-10 / 9}, \quad$ for $G<0.0111$

$R e_{\phi}>\left(\frac{1.85}{0.036}\right)^{4} G^{16 / 15}$, for $0.0111<G<0.0233$

$R e_{\phi}>\left(\frac{1.85}{0.0545}\right)^{10 / 3}$, for $G>0.0233$

In our case $R e_{\phi}$ is well beyond these critical values and, therefore, the flow is fully turbulent over the entire radial disk extension.

For a closed rotor-stator enclosure, the Daily-Nece correlations are

Regime III $C_{\mathrm{M}}=0.040 G^{-1 / 6} R_{\phi}^{-1 / 4}$

Regime IV $C_{\mathrm{M}}=0.051 G^{1 / 10} \operatorname{Re}_{\phi}^{-1 / 5}$

The corrected correlations proposed by Kreith [4, p. 177] are

Regime III $C_{\mathrm{M}}=0.036 G^{-1 / 6} R e_{\phi}^{-1 / 4}$

Regime IV $C_{\mathrm{M}}=0.0545 G^{1 / 10} \operatorname{Re}_{\phi}^{-1 / 5}$

The range of validity of these correlations is $R e_{\phi}<10^{7}$ and $0.0127<G<0.217$. Curiously, both original papers report the coefficients for Regime IV with a typographic error ( 0.0051 and 0.00545 , respectively).

In Regime III the boundary layers on the rotor and the stator are merged so that a continuous variation in tangential velocity exists across the gap. In Regime IV the combined thickness of the boundary layers on the rotor and the stator is less than the axial clearance $s$ so that between the two boundary layers there is a core region in which the tangential velocity has no axial variation.

For torque optimization purposes, it is noteworthy that in Regime III the moment coefficient is a relatively strongly decreasing function of gap clearance, whereas in Regime IV it is a slightly increasing function, that for large gap clearance tends to the free rotating disk limit (where Owen [1, p. 83] proposed the correlation $C_{\mathrm{M}}=$ $0.0655 R e_{\phi}^{-0.186}$, valid for $10^{5}<R e_{\phi}<10^{7}$, provided the flow is fully turbulent).

As a result, for a given value of $R e_{\phi}$ the minimum torque is attained at the transition between Regime III and Regime IV, i.e., for a gap clearance such that 
$G=\left(\frac{0.036}{0.0545}\right)^{15 / 4} R e_{\phi}^{-3 / 16}$

For our data, this optimum clearance corresponds to $G=$ 0.0132 at $300 \mathrm{~K}$ and $G=0.0145$ at $400 \mathrm{~K}$. Thus, we see that our case is already optimized with respect to torque.

However, the fact of being right on transition between Regimes III and IV requires further care in using this transition correlation. Indeed, for example a different optimal value of $G$ would have been obtained if, instead of the Kreith correlations, we adopted the Daily-Nece correlations. For this reason, we performed CFD simulations to verify the location of the optimal gap clearance. A reasonable agreement with the data predicted by the Kreith correlations has been found, as shown in Figs. $2(300 \mathrm{~K})$ and $3(400 \mathrm{~K})$. No curve fits of the Fluent points are provided in these figures because the precise optimal gap location has not been searched with the numerical simulations.

The agreement is less satisfactory when considering the power dissipation $W$, in that the difference between CFD simulation and the Kreith correlations is between $-3 \%$ and $-9 \%$.

These CFD simulations have been run for isothermal conditions because the correlations refer to data obtained for isothermal flow. The results of simulations for nonisothermal flow are discussed in Sections 3 and 4.

The transition between Regimes III and IV is well captured by the CFD simulations. Fig. 4 shows the dimensionless tangential velocity $v_{\phi} / \Omega r$ plotted as a function of distance from the rotating disk surface for four different axial gap clearances at the same radial location. For $G=0.025$ and 0.0357 the rotor and stator boundary layers are separated by a core region where the swirl does not vary axially (Regime IV). For $G=0.0143$, instead, the two boundary layers are clearly merged (Regime III) and for $G=0.00714$ they merge near their respective thicknesses (transition).

Using Fluent we solved the general mass, momentum and energy balance equations (see, for example, [14, Appendix A]), for steady axially symmetric flow with swirl. For turbulent flow over a rotating surface in axially symmetric flow the recommended turbulence model is a low Reynolds number $\kappa-\epsilon$ model developed by Morse [15]. In this respect, we adopted the RNG $\kappa-\epsilon$ model with the "swirl dominated flow" option and the "two-layer" model for near-wall treatment. This model requires a grid with at least 10 cells within the distance from the rotating surface where the wall-distance-based turbulent Reynolds number $R_{z}=z \sqrt{k_{\mathrm{T}}} / v<200\left(k_{\mathrm{T}}\right.$ is the turbulent kinetic energy).

In order to define the mesh according to this criterion, the line defined by the condition $R e_{z}=200$ can be estimated with a preliminary computation using the $\kappa-\epsilon$ model with standard wall functions and a relatively unrefined mesh. Once the $R e_{z}=200$ distance has been estimated, the final mesh can be obtained for example by grid refinement, as exemplified in Fig. 5. Alternatively, we can estimate the distance from the wall where $z^{+}=z \sqrt{\tau / \rho} / v=30$ based on the empirical correlations, place 12 cells within that distance, run the simulation and verify that there are at least 10 cells within $R e_{z}<$ 200. Grid accuracy outside the region where $R e_{z}<200$ was tested by successive mesh doubling.

It is noteworthy that in Regimes III and IV the effect of roughness on $C_{\mathrm{M}}$ becomes important if [1, p. 152]

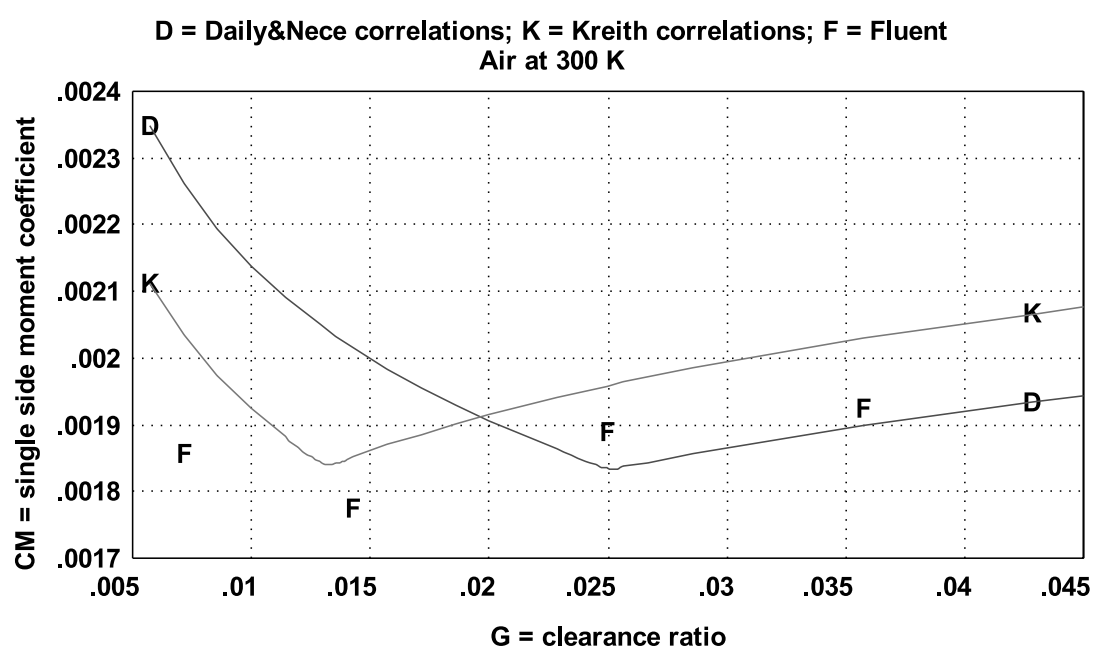

Fig. 2. Estimates of power dissipation. Comparison between Daily-Nece correlations, Kreith correlations and results of Fluent simulations. No radial flow. Isothermal flow at $300 \mathrm{~K} ; R e_{\phi}=2.63 \times 10^{6}$. 


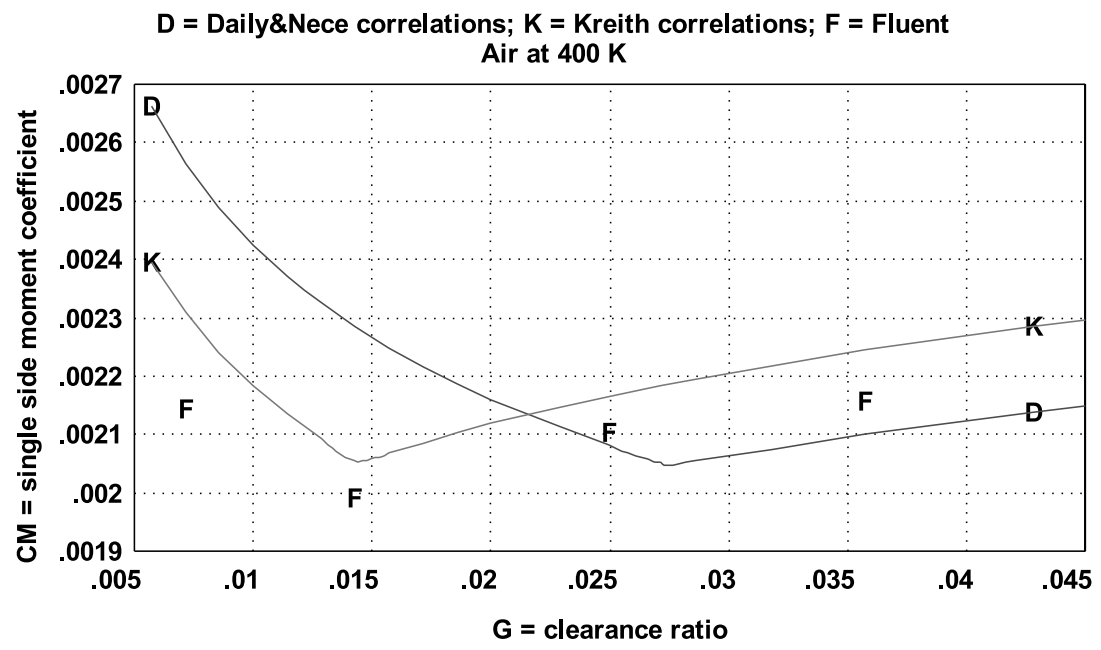

Fig. 3. Estimates of power dissipation. Comparison between Daily-Nece correlations, Kreith correlations and results of Fluent simulations. No radial flow. Isothermal flow at $400 \mathrm{~K} ; R e_{\phi}=1.595 \times 10^{6}$.

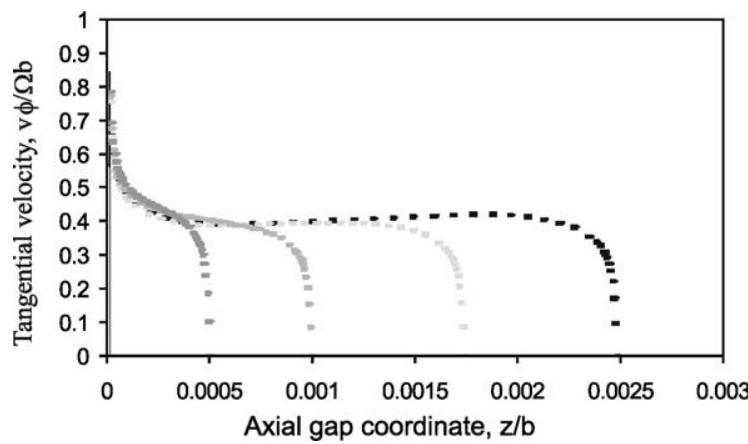

Fig. 4. Dimensionless swirl profiles across the gap for four different values of gap clearance.
$\frac{k_{\mathrm{s}}}{b}>\frac{180}{R e_{\phi}}$

where $k_{\mathrm{s}}$ is the effective height of roughness (for our data this means $\left.k_{\mathrm{s}}>4.5 \mu \mathrm{m}\right)$. When roughness is important, at high values of $R e_{\phi}$, Nece and Daily [16] found that $C_{\mathrm{M}}$ becomes almost independent of $R e_{\phi}$ and data are correlated by

$C_{\mathrm{M}}=-5.37 \log _{10}\left(k_{\mathrm{s}} / b\right)-3.4 G^{1 / 4}$

Finally it is noteworthy that power dissipation $W$ depends linearly on density (Eq. (6)) and, therefore, density reduction by application of some degree of vacuum can be effective in reducing $W$. However, it is shown in Sections 3 and 4 that moderate vacuum does not affect rotor temperatures in an adiabatic-rotor/isothermal-stator

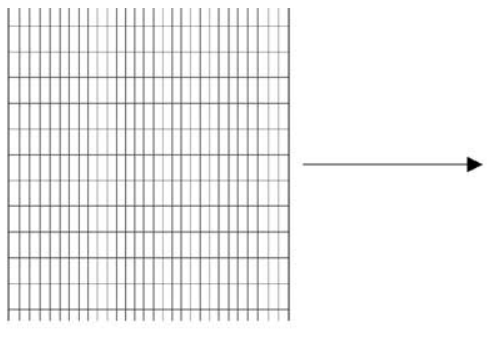

1) First grid 30 nodes between rotor and stator

RNG $k-\varepsilon$ Model

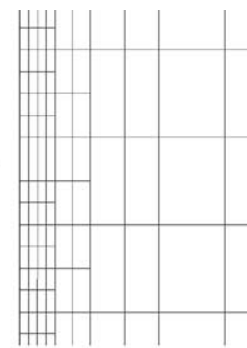

2) First boundary adapting

RNG $k-\varepsilon$ Model

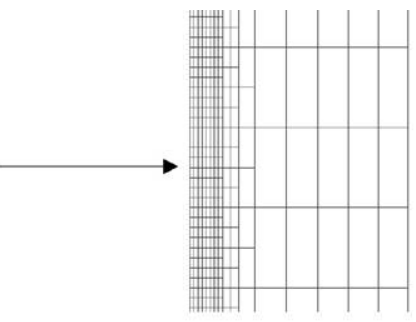

3) Second boundary adapting

Two-Layer Model

Fig. 5. Grid refinement. 
configuration (the Knudsen flow regime that may prevail under high-vacuum operation has not been investigated).

\section{Temperature estimates based on the Reynolds analogy}

For turbulent boundary layers on rotating surfaces, Dorfman, Owen and Rogers [14] and Chew [17] have proved the exact validity of the Reynolds analogy for flow within the boundary layer over a stationary or rotating surface, under the following broad set of conditions:

1. the boundary-layer assumptions are valid near the surface, i.e.:

- the gradients of all quantities, other than the pressure, have components normal to the surface much greater than the components parallel to the surface;

- the pressure gradient component normal to the surface is negligible;

- the flow velocity component normal to the surface is much smaller than the components parallel to the surface;

- the heat flux components normal to the surface is much greater than the components parallel to the surface;

2. the effective Prandtl number, $P r_{\text {eff }}=c_{\mathrm{p}} \mu_{\text {eff }} / k_{\text {eff }}$, must be unity, where $\mu_{\mathrm{eff}}$ is the ratio of the tangential shear stress component and the tangential velocity gradient in the direction normal to the surface, i.e., $\tau_{\phi z}=$ $\mu_{\text {eff }}\left(\partial v_{\phi} / \partial z\right)$, and $k_{\text {eff }}$ is the ratio of heat flux and temperature gradient components in the direction normal to the surface, i.e., $q_{z}=-k_{\text {eff }}(\partial T / \partial z)$;

3 . all boundary conditions must satisfy the same similarity condition between the total enthalpy $H=c_{\mathrm{p}} T+$ $v_{z}^{2} / 2+v_{\mathrm{r}}^{2} / 2+v_{\phi}^{2} / 2$ and the swirl $r v_{\phi}$, i.e.,

$H=C_{1} r v_{\phi}+C_{2}$

where $C_{1}$ and $C_{2}$ are the same constants for all boundary conditions.

When all these conditions are satisfied, it can be shown [14, p. 255] that the swirl $r v_{\phi}$ and the total enthalpy $H$ obey identical indefinite differential equations throughout the flow field and, by virtue of Condition 3 , the resulting solutions of these differential equations obey the similarity condition expressed in Eq. (18) throughout the entire flow field

$c_{\mathrm{p}} T+\frac{1}{2} v_{\mathrm{r}}^{2}+\frac{1}{2} v_{\phi}^{2}+\frac{1}{2} v_{z}^{2}=C_{1} r v_{\phi}+C_{2}$

It is noteworthy that this result includes dissipative as well as compressibility effects.
Differentiating Eq. (19) and using Condition 2 above, yields a relationship between tangential shear stress and normal heat flux valid anywhere in the flow field

$q_{z}-\mu_{\mathrm{eff}} v_{\mathrm{r}} \frac{\partial v_{\mathrm{r}}}{\partial z}-\mu_{\mathrm{eff}} v_{z} \frac{\partial v_{z}}{\partial z}=\left(v_{\phi}-C_{1} r\right) \tau_{\phi z}$

In almost all cases of interest to the present study, the rotor and stator boundary layers are merged to some extent. Therefore, the entire flow field is within one or the other boundary layer, i.e., the Reynolds analogy applies to the entire flow field in the gap between rotor and stator (provided, of course, that Conditions 2 and 3 are satisfied).

Eq. (19) on the stator surfaces, where $v_{\mathrm{r}}=v_{z}=$ $v_{\phi}=0$, requires

$C_{2}=c_{\mathrm{p}} T_{\mathrm{s}}$

which fixes the value of constant $C_{2}$ and means that the Reynolds analogy, in the presence of stationary surfaces, holds only if all these surfaces are isothermal at the same temperature.

On the rotor surfaces, where $v_{\mathrm{r}}=v_{z}=0$ and $v_{\phi}=\Omega r$, Eq. (19) requires

$c_{\mathrm{p}} T_{\mathrm{o}}+\frac{1}{2} \Omega^{2} r^{2}=C_{1} \Omega r^{2}+c_{\mathrm{p}} T_{\mathrm{s}}$

Moreover Eq. (20) at the boundaries implies

$q_{\mathrm{o}}=\left(\Omega-C_{1}\right) r \tau_{\mathrm{o}} \quad$ rotor surface

$q_{\mathrm{s}}=C_{1} r \tau_{\mathrm{s}} \quad$ stator surface

where, of course, $q_{\mathrm{o}}=\left.q_{z}\right|_{z=0}, q_{\mathrm{s}}=\left.q_{z}\right|_{z=s}, \tau_{\mathrm{o}}=\left.\tau_{\phi z}\right|_{z=0}$ and $\tau_{\mathrm{s}}=-\left.\tau_{\phi z}\right|_{z=s}$.

For adiabatic rotor surface, $q_{\mathrm{o}}=0$ implies

$C_{1}=\Omega$

and, therefore, the temperature distribution on the rotor surface is (Eq. (22))

$T_{\mathrm{o}}=T_{\mathrm{s}}+\frac{\Omega^{2} r^{2}}{2 c_{\mathrm{p}}}$

and anywhere in the flow field (Eq. (19)),

$c_{\mathrm{p}}\left(T-T_{\mathrm{s}}\right)+\frac{1}{2} v_{\mathrm{r}}^{2}+\frac{1}{2} v_{\phi}^{2}+\frac{1}{2} v_{z}^{2}=\Omega r v_{\phi}$

In particular, at the center of the gap $(z=s / 2)$

$T_{\mathrm{c}}=T_{\mathrm{s}}+\frac{\Omega^{2} r^{2}}{2 c_{\mathrm{p}}}\left(2 \beta-\beta^{2}-\frac{v_{\mathrm{rc}}^{2}}{\Omega^{2} r^{2}}-\frac{v_{z \mathrm{c}}^{2}}{\Omega^{2} r^{2}}\right)$

As suggested by Chew [17], for fluids with $\operatorname{Pr} \neq 1$ (and perhaps also for flows with $P r_{\text {eff }} \neq 1$ ) these results may be extended approximately by introducing a recovery factor, i.e., by the substitution 
$\frac{\Omega^{2}}{2 c_{\mathrm{p}}} \rightarrow \frac{\Omega^{2}}{2 c_{\mathrm{p}}} \operatorname{Pr}^{1 / 3} \quad$ or $\quad \frac{\Omega^{2}}{2 c_{\mathrm{p}}} \operatorname{Pr}_{\mathrm{eff}}^{1 / 3}$

As a means of verifying the numerical simulations against Eq. (27), we define the following dimensionless ratios

$\Theta=\frac{c_{\mathrm{p}}\left(T-T_{\mathrm{s}}\right)+\frac{1}{2} v_{\mathrm{r}}^{2}+\frac{1}{2} v_{\phi}^{2}+\frac{1}{2} v_{z}^{2}}{c_{\mathrm{p}}\left(T_{\mathrm{o}}-T_{\mathrm{s}}\right)+\frac{1}{2} \Omega^{2} r^{2}}$

$\Gamma=\frac{v_{\phi}}{\Omega r}$

which by virtue of Eq. (26) should be equal anywhere $(\Gamma=\Theta)$ in the gap.

\subsection{Validation for the closed enclosure case}

To validate the numerical simulations against the predictions of the Reynolds analogy, we considered the closed enclosure case with gap parameters already listed in Section 2.1. The value of the molecular $\operatorname{Pr}$ was set to unity by choosing the ad hoc value of $c_{\mathrm{p}}=1349 \mathrm{~J} / \mathrm{kg} \mathrm{K}$. However, the validation could only be approximate because we used the Fluent RNG $\kappa-\epsilon$ model which imposes a smooth variation of $P r_{\text {eff }}$ from the molecular value in the viscosity-dominated region to 0.85 in the fully turbulent region. For this reason, the recovery factor $\operatorname{Pr}_{\text {eff }}^{1 / 3}$ is used for purposes of comparison where appropriate.

Fig. 6 shows plots of $\Gamma, v_{\mathrm{r}} / \Omega b,\left(T-T_{\mathrm{s}}\right) /\left(T_{\mathrm{o}}-T_{\mathrm{s}}\right)$ and $(\Theta-\Gamma) / \Gamma$ versus $z / s$ across the gap at a radius $x=$

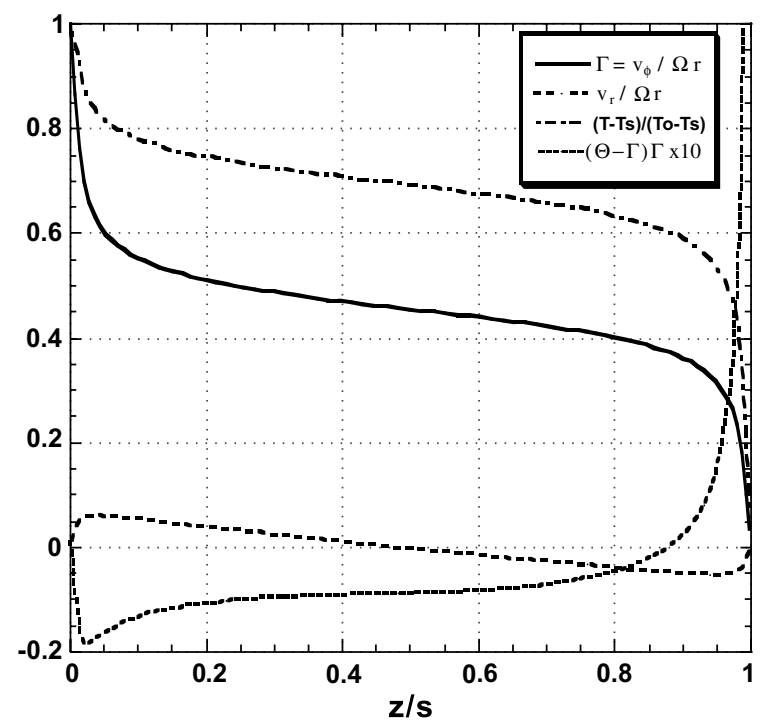

Fig. 6. Plots of $\Gamma=v_{\phi} / \Omega r, v_{\mathrm{r}} / \Omega r,\left(T-T_{\mathrm{s}}\right) /\left(T_{\mathrm{o}}-T_{\mathrm{s}}\right)$ and $(\Theta-\Gamma) / \Gamma \times 10$ versus $z / s$ across the gap at a radius $x=r / b=0.8$ for the four cases considered, solved with Fluent V4, with $R e_{\phi}=2.62 \times 10^{6}, G=0.0143, \operatorname{Pr}=1, \operatorname{Pr}_{\text {eff }}=0.85$, parabolic radial temperature imposed on rotor surface. $r / b=0.8$ for the closed enclosure case solved with Fluent V4, with rotor surface temperature imposed in accordance with the parabolic profile given by Eq. (26) with $C_{1}=\Omega$. The agreement is very good in that $(\Theta-\Gamma) / \Gamma$ is less than $2 \%$ except near the stator where $\Gamma$ goes to zero (note that $(\Theta-\Gamma) / \Gamma$ is magnified by a factor of 10). The balance between mechanical power dissipation $W$ and thermal power exchange $Q_{\mathrm{o}}+Q_{\mathrm{s}}$ at the rotor and stator is also satisfactory (within $2 \%$, evaluated by manual postprocessing). However, the rotor does not result adiabatic as the analogy would require, but a $13 \%$ contribution to the thermal power comes from the rotor. This disagreement with the requirements of the Reynolds analogy can also be seen from the plots of $q_{\mathrm{o}} / \tau_{\mathrm{o}} \Omega r$ and $q_{\mathrm{s}} / \tau_{\mathrm{s}} \Omega r$ versus $x=r / b$ shown in Fig. 7 (by virtue of the analogy, we should find $q_{\mathrm{o}} / \tau_{\mathrm{o}} \Omega r=0$ and $q_{\mathrm{s}} / \tau_{\mathrm{s}} \Omega r=1$ ).

For this reason, the closed case has been solved also with the adiabatic boundary condition on the rotor surface, both with Fluent V4 and Fluent V5.0.3 (which resulted in the more satisfactory energy unbalance of $1.4 \%)$. Fig. 8 shows plots of the dimensionless rotor temperature profiles

$\theta=\frac{T_{\mathrm{o}}-T_{\mathrm{s}}}{\Omega^{2} b^{2} / 2 c_{\mathrm{p}}}$

versus $x=r / b$, compared with the parabolic profile according to the Reynolds analogy (Eq. (26)), as well as with the correction suggested by Relation (29). The Fluent V4 results are closer to the parabolic profiles.

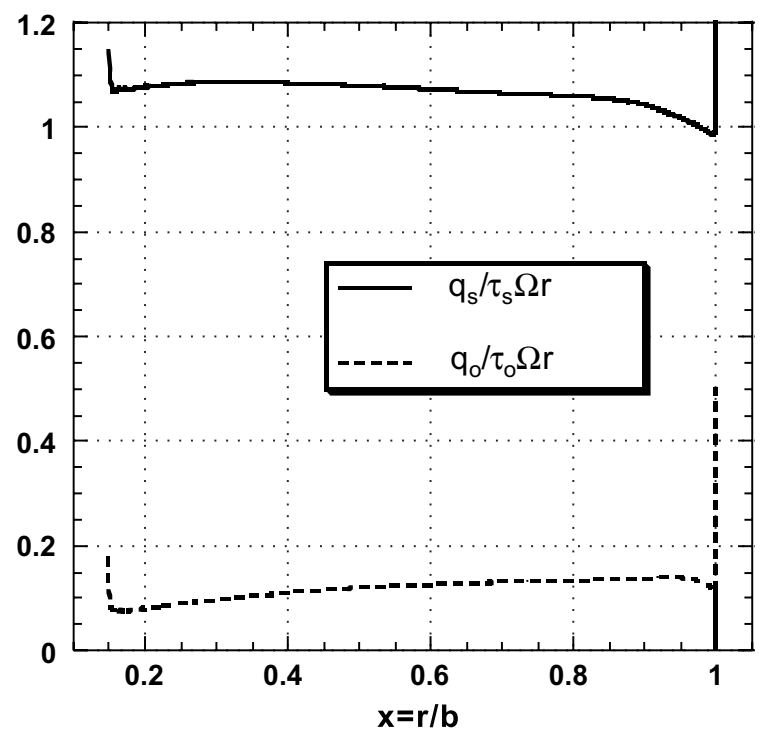

Fig. 7. Plots of $q_{\mathrm{o}} / \tau_{\mathrm{o}} \Omega r$ and $q_{\mathrm{s}} / \tau_{\mathrm{s}} \Omega r$ versus $x=r / b$ as obtained by postprocessing data from Fluent V4, same simulation as in Fig. 6. 


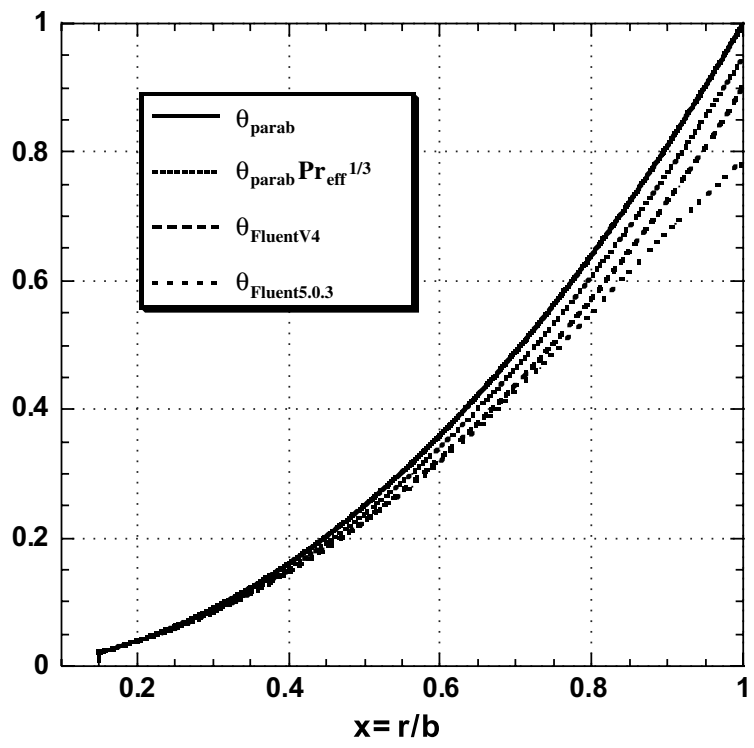

Fig. 8. Plots of dimensionless rotor temperature $\theta=2 c_{\mathrm{p}}\left(T_{\mathrm{o}}-\right.$ $\left.T_{\mathrm{s}}\right) / \Omega^{2} b^{2}$ versus $x=r / b$ for the closed enclosure case with adiabatic rotor condition solved with Fluent V4 and Fluent V5.0.3, with $\operatorname{Pr}=1, \operatorname{Pr}_{\text {eff }}=0.85, \operatorname{Re}_{\phi}=2.62 \times 10^{6}, G=0.0143 \mathrm{com}-$ pared with the parabolic profile according to the Reynolds analogy.

\subsection{Validation for open enclosure cases}

The Reynolds analogy applies also in presence of radial flow, as long as the conditions cited above are satisfied. To validate the numerical simulations against the predictions of the Reynolds analogy, we considered three cases: open $1 \mathrm{~mm}$, open $19 \mathrm{~mm}$, and open $38.6 \mathrm{~mm}$ with boundary conditions with suitable inlet swirl so as to satisfy Eq. (27). The agreement was good in all cases, however, due to space limitations and because such conditions are inadequate to represent the practical case, we do not report such results.

Instead, we consider here the open $38.6 \mathrm{~mm}$ with practical boundary conditions at both the inlet and the outlet sections set by imposing a "pressure inlet" condition (as implemented in Fluent V4 [18] and V5.0.3 [19]) with zero static pressure and an inlet temperature equal to the stator temperature $T_{\mathrm{s}}$, simulating the practical case of free inlet and outlet. These boundary conditions are not consistent with Eq. (27), therefore, the Reynolds analogy should not be expected to apply strictly, however, we compare anyway the results with the predictions of the analogy $(\Gamma=\Theta)$.

Fig. 9 shows plots of $\Gamma$ and $\Theta$ versus $z / s$ at $x=0.8$ (the inlet is on the stator surface open for $0 \leqslant x \leqslant$ $a / b=0.71), x=0.9$ and $x=1$ (outlet). The analogy between the developing swirl and temperature profiles is not strict, but still quite close.

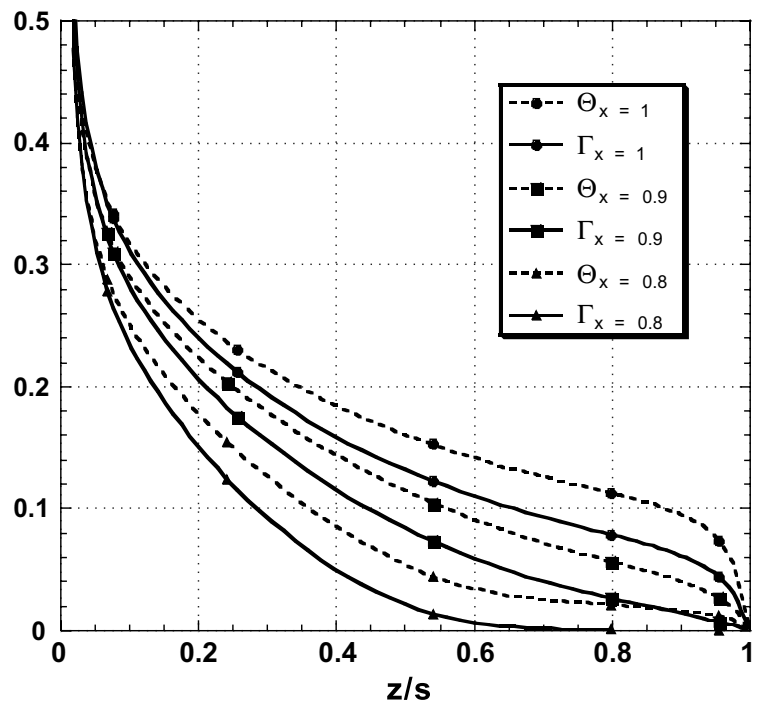

Fig. 9. Plots of $\Gamma$ and $\Theta$ versus $z / s$ at $x=0.8$ (inlet on stator surface at $x \leqslant a / b=0.71$ ), $x=0.9$ and $x=1$ (outlet) for the open $38.6 \mathrm{~mm}$ enclosure case with adiabatic rotor condition solved with Fluent V4, with $\operatorname{Pr}=1, \operatorname{Pr}_{\text {eff }}=0.85, R e_{\phi}=2.62 \times$ $10^{6}, G=0.0143$.

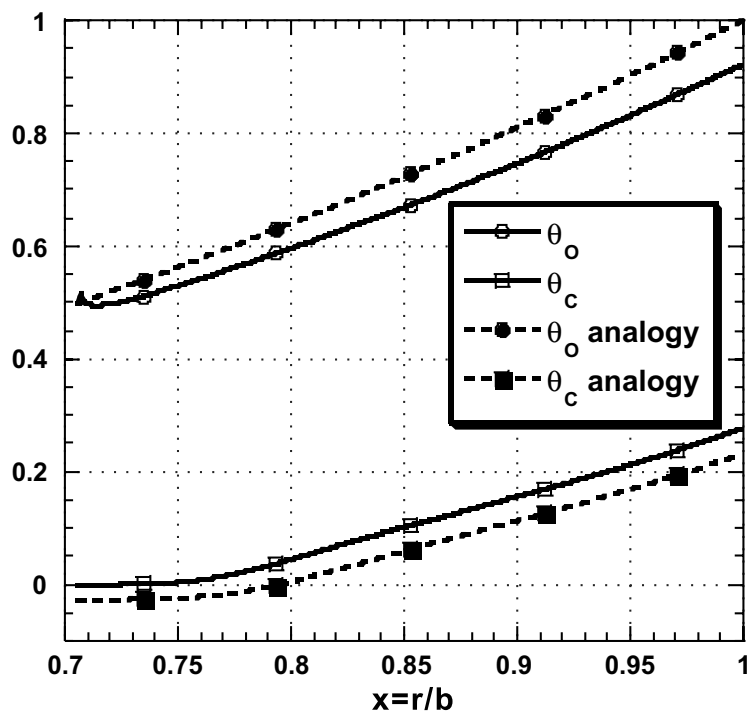

Fig. 10. Plots of rotor surface temperature, $T_{\mathrm{o}}$, and centergap temperature, $T_{\mathrm{c}}$, as functions of radial position $x=r / b$, for the open $38.6 \mathrm{~mm}$ enclosure case with adiabatic rotor condition solved with Fluent V4, and values of $T_{\mathrm{o}}$ and $T_{\mathrm{c}}$ given by Eqs. (26) and (28), respectively.

Fig. 10 shows the rotor surface temperature, $T_{\mathrm{o}}$, and the air temperature at the center of the gap, $T_{\mathrm{c}}$, as functions of $x=r / b$, and compares them with the respective predictions through the Reynolds analogy, 
given by Eqs. (26) and (28), yielding again a reasonable agreement, in spite of the fact that the boundary conditions are not strictly similar and that the boundary layers are rapidly developing.

Further analysis of this case is given later (Fig. 12).

\section{Model for flow field, moment coefficients and temper- atures}

In order to estimate the moment coefficient with or without radial flow in the gap, we developed a simple model based on an extension of that proposed in $[1, \mathrm{p}$. 162], on the assumption that the gap clearance is small and the rotor and stator boundary layers are merged.

We consider the gap clearance $s$ divided in two regions: the rotor boundary layer, $0<z<\gamma s$, and the stator boundary layer, $\gamma s<z<s$. We adopt a $1 / n$ power law approximation in both turbulent boundary layers

$$
\begin{aligned}
& \frac{\Omega r-v_{\phi}}{w_{\mathrm{o}}^{*}}=C_{\mathrm{n}}\left(\frac{z w_{\mathrm{o}}^{*}}{v}\right)^{1 / n} \\
& w_{\mathrm{o}}^{*}=\sqrt{\frac{\tau_{\mathrm{o}}}{\rho}} \text { for } 0<z<\gamma s \\
& \frac{v_{\phi}}{w_{\mathrm{s}}^{*}}=C_{\mathrm{n}}\left(\frac{(s-z) w_{\mathrm{s}}^{*}}{v}\right)^{1 / n} \\
& w_{\mathrm{s}}^{*}=\sqrt{\frac{\tau_{\mathrm{s}}}{\rho}} \text { for } \gamma s<z<s
\end{aligned}
$$

where, depending on the value of the appropriate Reynolds number (which is $\Omega r s / v=x R e_{\text {Couette }}$ ), the values of $n$ and $C_{\mathrm{n}}$ are: [20] $C_{7}=8.74, C_{8}=9.71, C_{9}=10.6$, $C_{10}=11.5$.

\subsection{No radial flow}

It can be shown [14, p. 265] that the two boundary layers have thicknesses proportional to the respective wall shear stresses. Therefore, we assume

$\gamma=\frac{\tau_{\mathrm{o}}}{\tau_{\mathrm{s}}+\tau_{\mathrm{o}}}$

Next we define

$\beta_{\gamma}=\frac{\left.v_{\phi}\right|_{z=\gamma s}}{\Omega r} \quad$ and $\quad \beta=\beta_{1 / 2}=\frac{\left.v_{\phi}\right|_{z=s / 2}}{\Omega r}$

and we note, for further reference, that the assumed swirl velocity profile implies that

$\tilde{v}_{\phi}=\frac{1}{s} \int_{\mathrm{o}}^{s} v_{\phi} \mathrm{d} z=\frac{\Omega r}{n+1}\left(\gamma+n \beta_{\gamma}\right)$

$1-\beta_{\gamma}=(1-\beta)(2 \gamma)^{1 / n}$
It is easy to show that Eqs. (33)-(35) yield the following expressions for the rotor and stator shear stresses

$\frac{\tau_{\mathrm{o}}}{\rho \Omega^{2} b^{2}}=C_{\mathrm{n}}^{-\frac{2 n}{n+1}} G^{-\frac{2}{n+1}} \operatorname{Re}_{\phi}^{-\frac{2}{n+1}} \gamma^{-\frac{2}{n+1}}\left(1-\beta_{\gamma}\right)^{\frac{2 n}{n+1}} x^{\frac{2 n}{n+1}}$

$\frac{\tau_{\mathrm{s}}}{\rho \Omega^{2} b^{2}}=C_{\mathrm{n}}^{-\frac{2 n}{n+1}} G^{-\frac{2}{n+1}} \operatorname{Re}_{\phi}^{-\frac{2}{n+1}}(1-\gamma)^{-\frac{2}{n+1}} \beta_{\gamma}^{\frac{2 n}{n+1}} x^{\frac{2 n}{n+1}}$

and the relation

$\frac{1-\gamma}{\gamma}=\left(\frac{\beta_{\gamma}}{1-\beta_{\gamma}}\right)^{\frac{2 n}{n+3}}$

By inserting Eq. (39) into (7) we may evaluate the moment coefficient

$C_{\mathrm{M}}=4 \pi C_{\mathrm{n}}^{-\frac{2 n}{n+1}} G^{-\frac{2}{n+1}} R e_{\phi}^{-\frac{2}{n+1}} \int_{x_{\mathrm{a}}}^{1} \gamma^{-\frac{2}{n+1}}\left(1-\beta_{\gamma}\right)^{\frac{2 n}{n+1}} x^{\frac{4 n+2}{n+1}} \mathrm{~d} x$

and in particular, for $n=7$,

$C_{\mathrm{M}}=0.28286 G^{-\frac{1}{4}} \operatorname{Re}_{\phi}^{-\frac{1}{4}} \int_{x_{\mathrm{a}}}^{1} \gamma^{-\frac{1}{4}}\left(1-\beta_{\gamma}\right)^{\frac{7}{4}} x^{\frac{15}{4}} \mathrm{~d} x$

It is noteworthy that the dependence on $R e_{\phi}$ is the same as in the Daily-Nece-Kreith correlation for Regime III (merged boundary layers). For the special case with $\gamma=\beta_{\gamma}=1 / 2$ and $x_{\mathrm{a}}=0$, which is a rough approximation for the closed enclosure case, this becomes

$C_{\mathrm{M}}=0.02105 G^{-\frac{1}{4}} R e_{\phi}^{-\frac{1}{4}}$

which, for $G=0.0143$ implies a $17 \%$ underestimate with respect to the Kreith correlation.

A better approximation for the closed enclosure case can be obtained by observing the plot (Fig. 11) of $\beta(x)$ obtained from the numerical simulation described in Fig. 6. The resulting average value of $\beta=0.44$ is in good agreement with a more elaborate analysis found in [1]. Assuming $\beta(x)$ from the numerical simulation, evaluating $\gamma(x)$ and $\beta_{\gamma}(x)$ by solving Eqs. (38) and (41) (results shown in Fig. 11), and the integral in Eq. (43), we find

$C_{\mathrm{M}}=0.02317 G^{-\frac{1}{4}} R e_{\phi}^{-\frac{1}{4}}$

which reduces the underestimate to $8 \%$.

\subsection{Cavities with radial flow}

For radial flow in the gap, the dependence of $\beta_{\gamma}$ and $\gamma$ on $r$ can be evaluated by means of an angular momentum balance

$\frac{\mathrm{d}}{\mathrm{d} r}\left(\dot{m} \overline{v_{\phi}} r\right)=2 \pi r^{2}\left(\tau_{\mathrm{o}}-\tau_{\mathrm{s}}\right)$ 


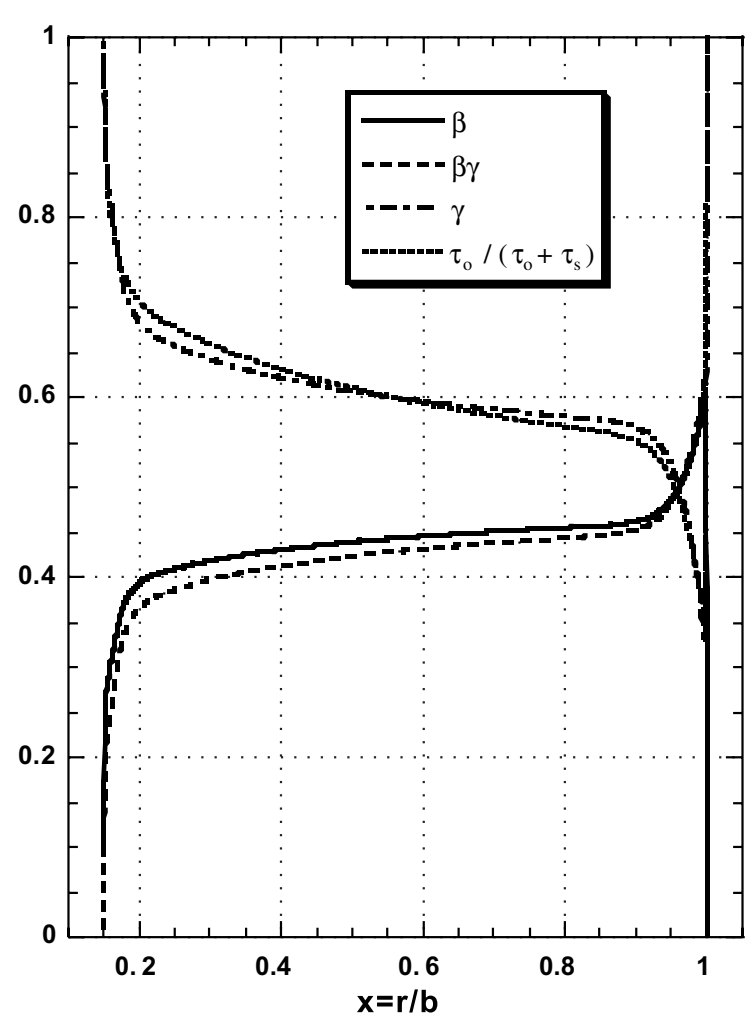

Fig. 11. Plots of $\beta(x)$ and $\tau_{\mathrm{o}}(x) /\left(\tau_{\mathrm{o}}(x)-\tau_{\mathrm{s}}(x)\right)$ for the closed enclosure case, solved with Fluent V4, with $R e_{\phi}=2.62 \times 10^{6}$ (based on average density $\bar{\rho}=1.153$ ), $G=0.0143, \operatorname{Pr}=1$, $P r_{\text {eff }}=0.85$, parabolic radial temperature imposed on the rotor surface. Values of $\gamma(x)$ and $\beta_{\gamma}(x)$ are obtained $\beta(x)$ by solving Eqs. (38) and (41). Moment coefficient: $C_{\mathrm{M}}=0.001817$ from Kreith correlation, $C_{\mathrm{M}}=0.001666$ from Eq. (45) $(-8 \%), C_{\mathrm{M}}=$ 0.001687 from Fluent data $(-7 \%)$.

where

$\dot{m}=2 \pi r \int_{0}^{s} \rho v_{\mathrm{r}} \mathrm{d} z$

$\overline{v_{\phi}}=\frac{2 \pi r}{\dot{m}} \int_{0}^{s} \rho v_{\mathrm{r}} v_{\phi} \mathrm{d} z$

and we neglect the net torque generated by the radial variation of the $\tau_{\mathrm{r} \phi}$ component of the stress tensor.

When $\dot{m}=$ const (i.e., neither injection nor suction through the rotor and stator surfaces), Eq (46) may be rewritten in dimensionless form

$\frac{C_{\mathrm{w}}}{2 \pi x^{2} R e_{\phi}}\left(\frac{\overline{v_{\phi}}}{\Omega b}+x \frac{\mathrm{d}}{\mathrm{d} x} \frac{\overline{v_{\phi}}}{\Omega b}\right)=\frac{\tau_{\mathrm{o}}}{\rho \Omega^{2} b^{2}}\left(1-\frac{\tau_{\mathrm{s}}}{\tau_{\mathrm{o}}}\right)$

where

$C_{\mathrm{w}}=\frac{\dot{m}}{\mu b}$
For substantial radial flow, we may use the approximation $\rho v_{\mathrm{r}} \approx$ const (i.e., flat profile) across the gap, to yield the approximation

$\overline{v_{\phi}} \approx \widetilde{v_{\phi}}=\frac{1}{s} \int_{\mathrm{o}}^{s} v_{\phi} \mathrm{d} z=\frac{\Omega b x}{n+1}\left(\gamma+n \beta_{\gamma}\right)$

Then, Eq. (46) may be rewritten as

$\frac{\mathrm{d} \beta_{\gamma}}{\mathrm{d} x}=\frac{A x^{\frac{2 n}{n+1}}}{C_{\mathrm{w}} D}-\frac{B}{D x}$

where

$A=2 \pi(n+1) C_{\mathrm{n}}^{-\frac{2 n}{n+1}} G^{-\frac{2}{n+1}} R e_{\phi}^{\frac{n-1}{n+1}}\left(1-\beta_{\gamma}\right)^{\frac{2 n}{n+1}} \gamma^{-\frac{2}{n+1}}\left(2-\frac{1}{\gamma}\right)$

$B=2\left(\gamma+n \beta_{\gamma}\right)$

$D=n\left[1-\frac{2}{n+3} \frac{\gamma^{2}}{\left(1-\beta_{\gamma}\right)^{2}}\left(\frac{1-\gamma}{\gamma}\right)^{\frac{n-3}{2 n}}\right]$

This differential equation, together with Eq. (41), can be readily solved numerically for given values of $n, G, R e_{\phi}$, $C_{\mathrm{w}}$ and $\left.\beta_{\gamma}\right|_{x=x_{\mathrm{a}}}$ (inlet swirl) to give the functions $\beta_{\gamma}(x)$, $\gamma(x)$ and $\beta(x)$ (from Eq. (38)), which in turn can be used in Eqs. (39), (40) and (7) to evaluate shear stresses and the moment coefficient, and in Eq. (28) to evaluate centergap temperatures, $T_{\mathrm{c}}(x)$.

The rotor surface temperature, instead, obeys Eq. (26) which is independent of the value of $C_{\mathrm{w}}$ and yields a reasonable estimate of $T_{\mathrm{o}}(x)$ also for boundary conditions inconsistent with the Reynolds analogy. This counterintuitive result is confirmed by Fig. 10, already discussed. Radial flow has a strong influence, instead, on the centergap temperature distribution $T_{\mathrm{c}}(x)$.

Fig. 12 shows the results of this procedure, for the case of radial flow considered in Figs. 9 and 10. The value of $C_{\mathrm{w}}$ is that obtained from the numerical simulation. The excellent agreement between $\beta_{\gamma}(x)$ and $\beta_{\text {Fluent }}(x)$, is lost when Eqs, (38) and (41) are used to evaluate $\beta(x)$. This is due to the fact that the $1 / n$ power law velocity profile, when extended from the rotor surface through the entire gap (i.e., when $\gamma=1$ and $\beta_{\gamma}=0$ ), implies that on the centerline $\beta=2^{-1 / n} \quad(=0.094$ for $n=7$ ), a value which is too high in the entrance region where the boundary layers are not merged and just beginning to develop, as already observed from Fig. 9.

Finally, we note that the approximation $\rho v_{\mathrm{r}} \approx$ const across the gap is poor in the limit of no radial flow $\left(C_{\mathrm{w}}=0\right)$, because it neglects the angular momentum transport associated with the secondary flows within the boundary layers (outward on the rotor and inward on the stator) due to the radial pressure gradient. Hence, 


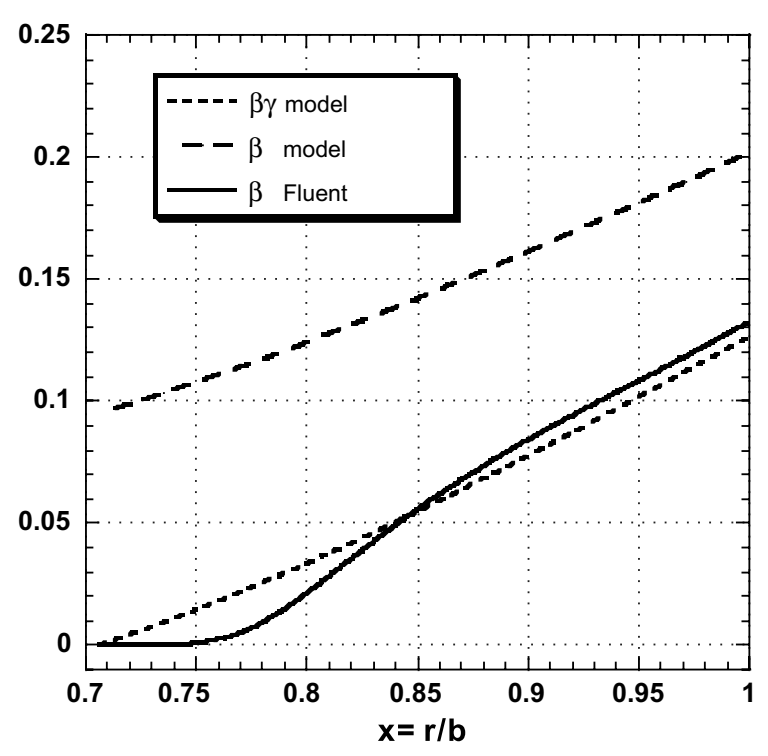

Fig. 12. Plots of $\beta_{\gamma}(x)$ and $\beta(x)$ calculated by solving Eq. (52) with $\operatorname{Re}_{\phi}=2.62 \times 10^{6}, G=0.0143, C_{\mathrm{w}}=23500, \beta_{\gamma}(0)=0$ and $n=7$, compared with the values of $\beta(x)$ obtained by FluentV4, same case as in Fig. 9.

the corresponding limiting solution $\beta_{\gamma}=\gamma=\beta=1 / 2$ and $\tau_{\mathrm{o}}=\tau_{\mathrm{s}}$ is a poor approximation.

\section{Conclusions}

We considered high-rotational-speed flow in rotorstator systems where viscous dissipation and compressibility effects generate important mechanical power dissipation and thermal load. The semi-empirical correlations available in the literature depend significantly upon flow regime and they are based on experimental data that do not extend to high-rotational Reynolds numbers. Therefore CFD computation can be used to provide additional data and attempt extensions of available correlations. However, great attention must be given to validating modeling assumptions and grid generation strategy.

Torque optimization by gap clearance adjustment is possible according to the available empirical correlations. This has been confirmed by numerical studies for the closed enclosure gap.

Modeling of the cavity between an adiabatic-rotor/ isothermal-stator configuration shows that the radial distribution of temperature difference between rotor and stator surfaces is parabolic. Under conditions of exact or approximate validity of the Reynolds analogy, it is independent of the presence and rate of radial flow. Thus, for practical conditions, to a reasonable degree of accuracy, radial flow:
1. reduces the thermal load of the stator, because much of the thermal power viscous generation within the rotor boundary layer is carried away by the radial flow itself instead of the heat conduction through the stator surface;

2. reduces centergap temperatures;

3. increases the moment coefficient, because much of the angular momentum gained by the flow in the rotor boundary layer is carried radially away and lost by the rotor-stator system;

4. does not reduce rotor surface temperature directly, however, it may reduce it indirectly if stator surface temperature turns out to be lower as a consequence of the reduced thermal load on the stator surface.

The rotor-stator temperature difference does not depend on fluid density, but depends only on the specific heat and only slightly on thermal conductivity and viscosity through the Prandtl number. Thus, density reduction will reduce power dissipation but not rotor surface temperature.

\section{References}

[1] J.M. Owen, R.H. Rogers, Flow and Heat Transfer in Rotating-Disk Systems, vol. 1: Rotor-Stator Systems, Wiley, New York, 1989.

[2] R.E. Mayle, C. Hirsch, S. Hess, J. von Wolfersdorf, Rotorstator gap flow analysis and experiments, IEEE Trans. Energy Conver. 13 (June) (1998) 101-110.

[3] J.W. Daily, R.E. Nece, Chamber dimensions effects on induced flow and frictional resistance of enclosed rotating disks, Trans. ASME, J. Basic Eng. 82 (March) (1960) 217232.

[4] F. Kreith, Convection heat transfer in rotating systems, Adv. Heat Transfer 5 (1968) 129-251.

[5] J.M. Owen, Fluid flow and heat transfer in rotating disc systems, in: D.E. Metzger, N.H. Afgan (Eds.), Heat and Mass Transfer in Rotating Machinery, Hemisphere publishing corporation, Washington, 1984, pp. 81-103.

[6] A. Northrop, J.M. Owen, Heat transfer measurements in rotating-disk systems. Part 1: The free disc, Int. J. Heat Fluid Flow 9 (1) (1988) 19-26.

[7] A. Northrop, J.M. Owen, Heat transfer measurements in rotating-disc systems. Part 2: The rotating cavity with a radial outflow of cooling air, Int. J. Heat Fluid Flow 9 (1) (1988) 27-38.

[8] D.M. Maron, S. Cohen, Hydrodynamics and heat/mass transfer near rotating surfaces, Adv. Heat Transfer 21 (1991) 141-183.

[9] C.L. Ong, J.M. Owen, Computation of the flow and heat transfer due to a rotating disc, Int. J. Heat Fluid Flow 12 (2) (1991) 106-115.

[10] C.L. Ong, J.M. Owen, Prediction of heat transfer in a rotating cavity with a radial outflow, Trans. ASME, J. Turbomach. 113 (January) (1991) 115-122.

[11] J.X. Chen, X. Gan, J.M. Owen, Flow and heat transfer in enclosed rotor-stator systems, in: G.F. Hewitt (Ed.), 
Proceedings of the 10th International Heat Transfer Conference, Brighton, IChemE, 1994, pp. 161-166.

[12] J.X. Chen, X. Gan, J.M. Owen, Heat transfer in an aircooled rotor-stator system, Trans. ASME, J. Turbomach. 118 (July) (1996) 444-451.

[13] J.X. Chen, X. Gan, J.M. Owen, Heat transfer from aircooled contrarotating disks, Trans. ASME, J. Turbomach. 119 (January) (1997) 61-67.

[14] J.M. Owen, R.H. Rogers, Flow and Heat Transfer in Rotating-Disk Systems, vol. 2: Rotating Cavities, Wiley, New York, 1995.

[15] A.P. Morse, Numerical prediction of turbulent flow in rotating cavities, Trans. ASME, J. Turbomach. 110 (April) (1988) 202-212.
[16] R.E. Nece, J.W. Daily, Roughness effects on frictional resistance of enclosed rotating disks, Trans. ASME, J. Basic Eng. 82 (1960) 553-562.

[17] J. Chew, Effect of frictional heating and compressive work in rotating axisymmetric flow, Trans. ASME, J. Heat Transfer 107 (November) (1985) 984-986.

[18] Fluent V4. Pressure inlet boundary conditions. In User's Guide, Fluent Inc., Lebabon, NH, USA, 1996, pp. 14.5814.89.

[19] Fluent V5. Pressure inlet boundary conditions. In User's Guide, Fluent Inc., Lebabon, NH, USA, 1998, pp. 6.176.27 .

[20] H. Schlichting, Boundary-Layer Theory, McGraw-Hill, New York, 1979. 